\title{
A simplified laboratory validated assay for MGMT promoter hypermethylation analysis of glioma specimens from formalin-fixed paraffin-embedded tissue
}

\author{
Milena Cankovic ${ }^{1}$, Tom Mikkelsen², Mark L Rosenblum² and Richard J Zarbo
}

Glioma, and in particular high-grade astrocytoma termed glioblastoma multiforme (GBM), is the most common primary tumor of the brain. Epigenetic silencing of the MGMT $\left(\mathrm{O}^{6}\right.$-methylguanine-DNA Methyl transferase) DNA repair gene by promoter methylation compromises DNA repair and has been associated with longer survival in patients with GBM who receive alkylating agents. The methylation status of the MGMT promoter is determined by methylation-specific polymerase chain reaction analysis (MSP). This protocol is often challenging with GBM specimens, because of extensive necrosis and scarcity of malignant cells. The objective of this study was to develop a reliable, clinically validated assay for detection of epigenetic silencing of the MGMT gene using formalin-fixed, paraffin-embedded brain tumor resections and methylation-specific PCR.

Laboratory Investigation (2007) 87, 392-397. doi:10.1038/labinvest.3700520; published online 29 January 2007

KEYWORDS: formalin-fixed tissue; glioblastoma multiforme; methylation-specific PCR; MGMT; promoter hypermethylation; Temozolomide

Gliomas are the most common tumors of the brain. They can be classified histologically as astrocytomas, oligodendrogliomas, or tumors with morphological features of both astrocytes and oligodendrocytes. According to the degree of malignancy, gliomas are divided into four clinical grades, with grades I and II representing benign tumors, and grade III and IV representing the malignant tumors. Grade IV gliomas, also called glioblastoma multiforme (GBM), account for $30 \%$ of primary brain tumors in adults. ${ }^{1}$ Patients with glioblastoma multiforme have a mean survival of about 12 months. Chemotherapy for GBM has very limited efficacy, however, it has been shown that certain patients may respond to some treatments. ${ }^{2,3}$ Temozolomide is a novel alkylating agent that has demonstrated activity in recurrent gliomas. ${ }^{4-6}$ Alkylating agents cause cell death by forming cross-links between adjacent strands of DNA due to alkylation of the $\mathrm{O}^{6}$ position of guanine. The cellular DNA repair protein $\mathrm{O}^{6}$ methylguanine-DNA methyltransferase (MGMT) functions as a DNA repair enzyme that removes the mutagenic alkyladducts from the $\mathrm{O}^{6}$-poasition of guanine and thereby causes resistance to alkylating drugs. ${ }^{7-9}$ Tumors appear to be het- erogeneous with respect to MGMT expression, and in a subset of cancer cells, its expression is silenced due to abnormal promoter methylation. Aberrant methylation of CpG islands located in the promoter region of MGMT gene is associated with transcriptional inactivation of this DNA repair gene, and consequent low levels of the DNA repair enzyme. Studies have shown that patients with low levels of this DNA repair enzyme are more likely to experience response to therapy and prolonged overall and disease-free survival. ${ }^{10-13}$

Aberrant methylation of $\mathrm{CpG}$ islands in the promoter region of many genes has been recognized as an important epigenetic mechanism for gene silencing. ${ }^{14-17}$ Inactivation of multiple tumor suppressor genes by aberrant hypermethylation is a fundamental process involved in the development of many malignant tumors. ${ }^{18,19}$ Mapping of methylation patterns in CpG islands has become an important tool in understanding tissue-specific gene expression in both normal and pathologic situations, and several protocols have been published for evaluating methylation status by methylationspecific PCR (MSP). ${ }^{20,21}$ These protocols are based on bisulfite treatment of isolated DNA. Bisulfite treatment 
chemically changes unmethylated, but not methylated, cytosines to uracil. The methylated and unmethylated sequences are detected through the use of methylation-specific primers.

MGMT MSP protocols described in the literature use frozen tissue, or combination of frozen and formalin-fixed paraffin-embedded tissue (FFPE), and call for $2-3 \mu \mathrm{g}$ of isolated DNA. This presents a challenge because only archival FFPE tissue is available in many pathology practices, and when these preserved specimens are used for testing of gliomas, and especially glioblastoma multiforme, quite frequently a very small amount of sometimes partially degraded DNA is recovered due to extensive necrosis and scarcity of malignant cells. Our aim was to develop a reliable, simplified MGMT MSP protocol that would utilize FFPE specimens and thus could be easily incorporated into a busy molecular diagnostics laboratory practice. Our protocol is a slight modification of the protocol originally published by Esteller et $a l,{ }^{20}$ and involves the use of thick tissue sections and bisulfite treatment and purification of treated DNA with the help of commercial methylation kits.

\section{MATERIALS AND METHODS Tumor Samples}

A total of 167 brain excision specimens from 154 patients were submitted for MGMT MSP testing. Pathologic diagnoses included 74 patients with glioblastoma (44 male subjects, 30 female subjects, mean age $55.62 \pm 12.88$ years, range 34-82); 63 patients with oligodendrogliomas and mixed tumors (36 male subjects and 27 female subjects, mean age $41.66 \pm 12.42$ years, range $18-74)$; and 17 patients with astrocytoma (13 male subjects and four female subjects, mean age $37.39 \pm 13.27$ years, range $20-71)$. The specimens had been collected from 1997 to 2006, the majority being more recent specimens. The study group included primary tumors and recurring tumors. Paired primary and recurrent specimens were available for ten patients. All were submitted as formalin fixed paraffin embedded tissue blocks, and were grouped by the following tumor types: 82 cases of glioblastoma multiforme (GBM), seven cases of astrocytoma (A), eight cases of anaplastic astrocytoma (AA), 31 cases of oligodendroglioma $(\mathrm{O})$ and 30 cases of anaplastic oligodendroglioma (AO). Additional mixed tumor cases consisted of four cases of $\mathrm{AOA}$, three cases of $\mathrm{OA}$, one case of $\mathrm{O}+\mathrm{AA}$, and one case of malignant glioneuronal neoplasm. For all tumor samples, histology was reviewed to select blocks with greatest tumor involvement and smallest amount of necrosis. In addition to tumors, 11 samples were studied of cortex blocks from epilepsy resections.

\section{DNA Extraction and Methylation-Specific PCR}

Genomic DNA was isolated from two to three $20 \mu \mathrm{M}$ thick paraffin sections after confirmation of the histology. DNA from FFPE tissue was extracted using Puregene kit (Gentra Systems, Minneapolis, MN, USA). The MSP was performed in a two step approach. Bisulfite treatment of isolated DNA was done using EZ DNA Methylation Gold kit (Zymo Research, Orange, CA, USA). PCR amplification was performed basically as reported by Esteller et $a l^{20}$ with specific primers designed to distinguish methylated from unmethylated DNA. The primer sequences used were as follows: $\mathrm{M}$ forward; $5^{\prime}$ TTTCGACGTTCGTAGGTTTTCGC 3', M reverse; 5' GCA CTCTTCCGAAAACGAAACG $3^{\prime}$; U forward; $5^{\prime}$ TTTGTGT TTTGATGTTGTTAGGTTTTTGT $3^{\prime}$, and $\mathrm{U}$ reverse; $5^{\prime}$ AA CTCCACACTCTTCCAAAAACAAAACA $3^{\prime}$.

For assay controls, methylated DNA and unmethylated DNA were purchased from Chemicon International, Temecula, CA, USA, and used as positive and negative controls for methylated sequences, respectively. The control DNA was subjected to bisulfite treatment and PCR amplification in parallel with patient samples for every run. Controls without DNA were performed for each set of reactions.

Methylated and unmethylated DNA sequences were detected on $2.5 \%$ agarose gels. Samples giving signals approximately equivalent to the positive methylated control were designated as methylated. Samples giving no signals with positive methylated control, but demonstrating presence of unmethylated DNA, similar to the negative control, were designated as unmethylated. The results were confirmed in an independent experiment on 10 randomly selected specimens, starting with reisolation of DNA from the tumor samples. The investigators who analyzed the glioma samples were blinded to all clinical information.

\section{Immunohistochemistry for MGMT}

Parallel immunohistochemical staining of the MGMT protein product was done on a subset of the study cases (11 methylation positive cases, and 11 methylation-negative cases). Immunohistochemical analysis was performed on formalin fixed, paraffin embedded tissue sections. Immunostaining was performed using heat-induced epitope retrieval, a labeled streptavidin-biotin method, and an automated immunostainer (DAKO, Carpinteria, CA, USA) according to the manufacturer's protocol. Primary antibody against MGMT (Mouse anti-MGMT, clone MT 23.2, Invitrogen, Carlsbad, CA, USA) was used at 1:75 dilution. Immunohistochemistry was evaluated by two independent observers who were blinded from methylation data. MGMT expression of malignant cells was interpreted as negative when $0-5 \%$ of tumor cells showed nuclear staining, and positive when $>5 \%$ of tumor cells stained positive for MGMT. Only nuclear staining was considered for evaluation.

\section{RESULTS}

Of the 167 evaluated tumors, MGMT promoter methylation status was determined for 164 specimens (98\%). Table 1 shows a summary of the findings from MSP for the entire study group. A representative methylation specific PCR of MGMT promoter in gliomas is shown in Figure 1. Overall, methylation specific PCR identified $72(44 \%)$ specimens in 
Table 1 Summary of MGMT MSP Results

\begin{tabular}{|c|c|c|c|c|c|}
\hline Diagnosis & WHO grade & $\begin{array}{l}\text { Age mean } \pm \text { s.d. } \\
\text { (range) }\end{array}$ & $\begin{array}{l}\text { No. of specimens } \\
\text { tested (\%) }\end{array}$ & $\begin{array}{l}\text { Methylated } M G M T \\
\text { promoter (\%) }\end{array}$ & $\begin{array}{c}\text { Unmethylated } M G M T \\
\text { promoter (\%) }\end{array}$ \\
\hline A & $\|$ & $38.74 \pm 17.41(20-71)$ & 6 & $2(33)$ & $4(67)$ \\
\hline AA & III & $37.25 \pm 12.56(20-55)$ & 8 & $3(38)$ & $5(62)$ \\
\hline $\mathrm{AOA}$ & III & $43.5 \pm 6.81(35-50)$ & 4 & 2 & 2 \\
\hline $\mathrm{OA}$ & $\|$ & $43 \pm 7.94(35-49)$ & 3 & 1 & 2 \\
\hline $\mathrm{O}+\mathrm{AA}$ & III & (35) & 1 & 1 & 0 \\
\hline MGN & & (23) & 1 & 1 & 0 \\
\hline
\end{tabular}

Abbreviations: GBM: glioblastoma multiforme; A: astrocytoma; AA: anaplastic astrocytoma, O: oligodendroglioma; AO: anaplastic oligodendroglioma; AOA: mixed anaplastic oligodendroglioma + astrocytoma; OA: oligodendroglioma + astrocytoma; MGN: malignant glioneuronal neoplasm.

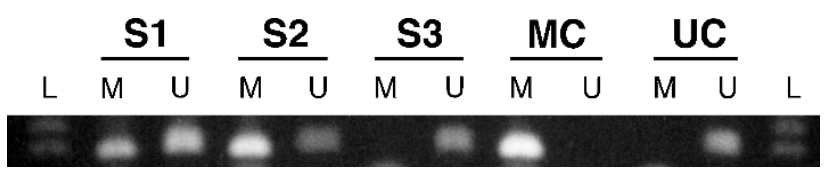

Figure 1 Methylation specific PCR of MGMT promoter in gliomas. Three different brain tumor samples were tested. Two samples were methylated, although they showed both methylated and unmethylated bands. One sample was unmethylated. M, PCR product amplified by methylatedspecific primers; U, PCR product amplified by unmethylated-specific primers; L, ladder; MC, methylated control DNA; UC, unmethylated control DNA.

which methylated MGMT promoter was present and 92 (56\%) of tumors in which only unmethylated MGMT promoter was detectable. The methylation frequencies differed among tumor subtypes, although those differences were not always statistically significant. Low-grade (WHO grade II) oligodendrogliomas and astrocytomas displayed lower methylation rates (26 and 33\%, respectively), than anaplastic (WHO grade III) tumors (63\% for $\mathrm{AO}$ and $38 \%$ for $\mathrm{AA}$ ), suggesting a trend towards increase in methylation with higher tumor grades. That trend, however, did not continue for grade IV tumors. The glioblastoma group, which consisted of primary and secondary tumors, had a methylation rate of $44 \%$. In the control group, all 11 epilepsy resection samples displayed ummethylated alleles for MGMT. Paired primary and recurring specimens were available for $10 \mathrm{pa}-$ tients (Table 2). In five of the patients, no evidence of MGMT promoter methylation was detected in either primary or recurrent tumor specimens. Two of the primary tumors gave positive results, while the recurrent sections had no evidence of MGMT methylation. For two of the patients, both primary and secondary tumors had evidence of promoter methyla-
Table 2 Paired primary and repeat resections

\begin{tabular}{lclcl}
\hline $\begin{array}{l}\text { Case } \\
\text { number }\end{array}$ & $\begin{array}{c}\text { Initial surgery } \\
\text { date }\end{array}$ & MSP result & $\begin{array}{c}\text { Repeat surgery } \\
\text { date }\end{array}$ & MSP result \\
\hline 1 & $9 / 24 / 2004$ & Unmethylated & $11 / 7 / 2005$ & Unmethylated \\
2 & $12 / 19 / 2000$ & Unmethylated & $12 / 20 / 2004$ & Unmethylated \\
3 & $8 / 1 / 1997$ & Unmethylated & $2 / 27 / 1998$ & Unmethylated \\
4 & $2 / 10 / 2006$ & Methylated & $5 / 19 / 2006$ & Methylated \\
5 & $8 / 4 / 2003$ & Methylated & $12 / 15 / 2004$ & Unmethylated \\
6 & $6 / 13 / 2005$ & Unmethylated & $12 / 21 / 2005$ & Unmethylated \\
7 & $4 / 6 / 2004$ & Unmethylated & $11 / 28 / 2005$ & Unmethylated \\
8 & $7 / 27 / 2000$ & Unmethylated & $4 / 25 / 2005$ & Methylated \\
9 & $8 / 1 / 2005$ & Methylated & $8 / 4 / 2005$ & Methylated \\
10 & $3 / 1 / 1999$ & Methylated & $11 / 17 / 1999$ & Unmethylated \\
\hline
\end{tabular}

tion. Finally, one case presented with no evidence of methylated MGMT promoter in primary tumor, while the methylated promoter was detected in the recurrent tumor.

To evaluate whether MGMT promoter hypermethylation differed between male and female patients, we compared the MSP results for male and female patients for glioblastoma multiforme and oligodendroglioma groups (Table 3). In the GBM group, the male patients were significantly older (mean age 57.39 years) compared to female subjects (mean age 39 years), and the younger female group demonstrated a higher methylation rate. With male subjects, methylated MGMT promoter was detected in 20/49 (41\%) GMB specimens, and with female subjects, the methylation rate was $15 / 31$ (48\%) of specimens. In the oligodendroglioma group, mean ages for male and female were 39 and 43 years, respectively, however, 
Table 3 GBM and oligodendroglioma MSP results separated by gender

\begin{tabular}{|c|c|c|c|c|c|}
\hline GBM males & 44 & $57.39 \pm 10.93(40-76)$ & 49 & $20(41)$ & $29(59)$ \\
\hline O males & 18 & $39.06 \pm 10.33(20-62)$ & 18 & $4(22)$ & $14(78)$ \\
\hline O females & 12 & $43 \pm 15.52(18-60)$ & 13 & $4(31)$ & $9(69)$ \\
\hline AO females & 15 & $43.06 \pm 15.11(18-68)$ & 16 & $10(63)$ & $6(37)$ \\
\hline
\end{tabular}

Abbreviations: GBM: glioblastoma multiforme; O: oligodendroglioma; AO: anaplastic oligodendroglioma.

significant difference in methylation rates was observed: $4 / 18$ (22\%) for male subjects and 4/13 (31\%) for female subjects. Finally, in the anaplastic oligodendroglioma group, there was no difference between the two groups, with methylation rates of $9 / 14(64 \%)$ for male subjects and 10/16 (63\%) for female subjects.

For all brain tumor resection cases, MGMT promoter hypermethylation was always accompanied by amplification in the unmethylated reaction as well. This is to be expected since the original tissue sections contained a mixture of tumor and non-malignant tissue. Similar findings were reported previously in CpG island methylation studies. ${ }^{20,22,23}$ Actually, the presence of unmethylated promoter served as an internal amplification control that could be used to assess the quality and quantity of DNA. Only tumor samples that contained a clearly visible methylated signal, with or without an additional unmethylated signal, were interpreted as positive for the MGMT promoter methylation.

\section{Immunohistochemical Findings}

We compared promoter methylation status to loss of protein expression as assessed by immunohistochemistry. This aspect of the study allowed a comparative analysis of the results of the MGMT MSP and assessment of the predictive value of MGMT promoter methylation (as determined by MGMT MSP) for loss of protein expression. Intact protein expression was observed in $9 / 11(82 \%)$ of methylation-negative cases. Promoter methylation was associated with loss of protein expression in $8 / 11(73 \%)$ of cases. In all positive tumor samples, heterogeneous immunostaining was observed (Figure 2), where areas with complete loss of MGMT expression alternated with areas of scattered or clustered cells with strong immunoreactivity.

\section{DISCUSSION}

We studied a heterogeneous group of 154 glioma patients for the evidence of MGMT promoter hypermethylation by methylation specific PCR. Of the 164 specimens evaluated, 72 (44\%) had a detectable methylated MGMT promoter, whereas $92(56 \%)$ did not (Table 1$)$. The promoter methy- lation status appeared to differ between different sub-groups, similar to what has been reported previously. ${ }^{13,24,25}$ In this study, the glioblastoma group, which consisted of primary and secondary tumors, presented a methylation rate of $44 \%$. This is concordant with the results of Hegi et al ${ }^{4}$ who reported a methylation rate of $44.7 \%$ of 206 glioblastoma multiforme specimens, and Esteller et al, ${ }^{12}$ who studied a group of 47 patients with grade II and IV gliomas and detected MGMT promoter hypermethylation in 19 of the 47 tumors $(40 \%)$. In the oligodendroglioma group, our results are discordant with those of Möllemann et $a l^{26}$ who investigated 52 oligodendroglial tumors and reported a methylation rate of $88 \%$, whereas we obtained a rate of $63 \%$ in 30 anaplastic oligodendroglioma cases in our study (Table 1). One limitation of this study is the lack of information about clinical outcomes, which might have corroborated possible differences in responses between groups.

DNA methylation-dependent silencing of gene expression in cancer results in loss of protein expression and consequently protein function. In order to evaluate correlation between MGMT promoter methylation and loss of protein expression in our study group, a subset of 22 cases were evaluated for MGMT protein expression by immunohistochemistry. In this small group of 22 cases (11 methylation positive, and 11 methylation-negative), MGMT promoter methylation was associated with loss of protein expression. In positive tumor samples, heterogeneous immunostaining was observed, similarly to previous observations. ${ }^{12,26}$ In positive cases, total number of MGMT positive cells was generally fewer than $50 \%$ of total tumor cells. There were a few cases, however, that demonstrated loss of expression without promoter methylation, and also cases with intact protein expression despite promoter methylation. Similar observations for MGMT expression were reported by Brell et $\mathrm{al}^{13}$ for a group of 93 cases of anaplastic glioma, and Ogino et $a l^{27}$ who studied correlation between CDKN2A, MLH1 and MGMT promoter methylation and protein loss by immunohistochemistry in a much larger group of 274 colorectal cancer cases. ${ }^{27}$ Mechanisms such as gene deletion or mutation have been implicated as alternative mechanisms 

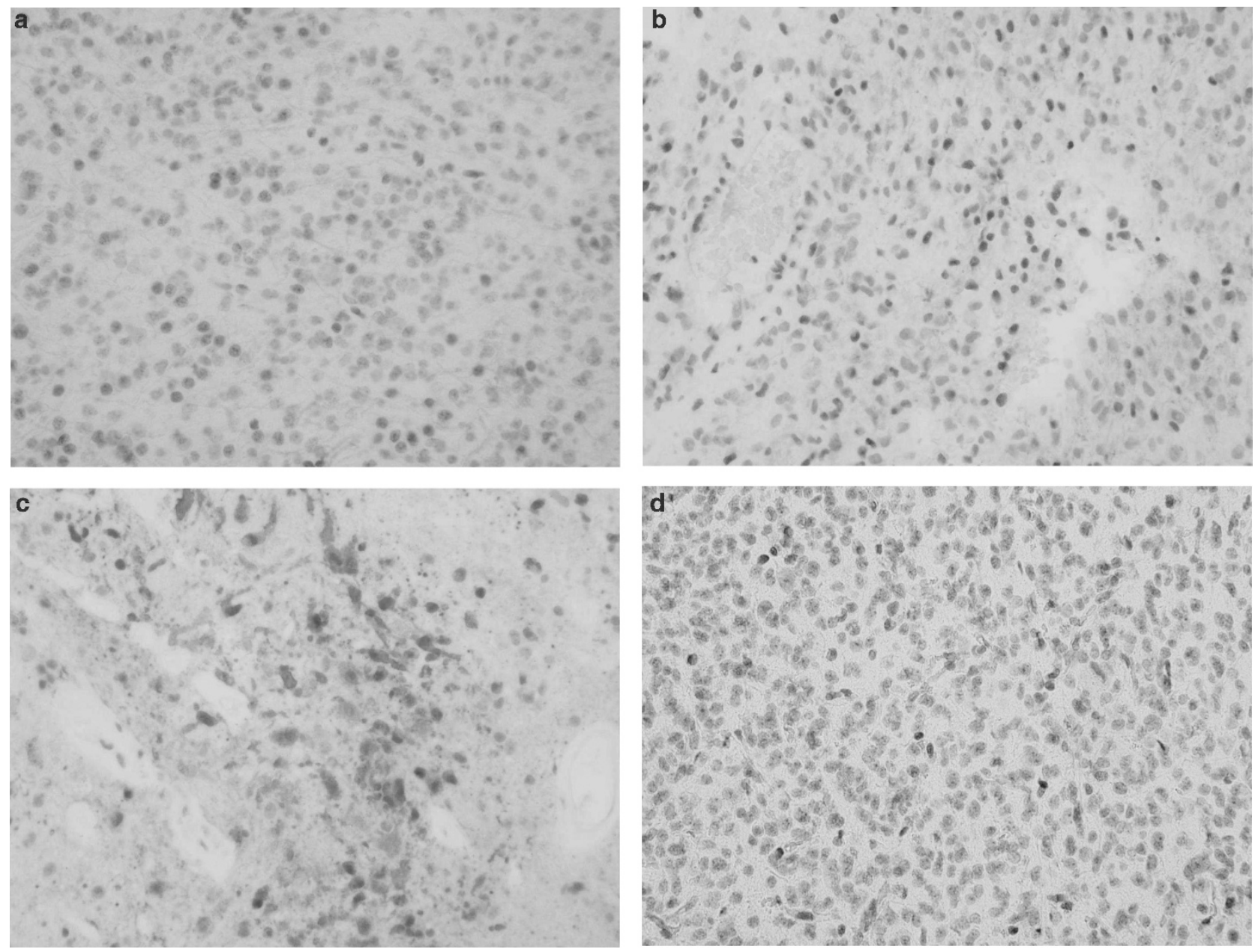

Figure 2 Immunohistochemistry of MGMT gene product. (a) An oligodendroglioma case and (b-c) two glioblastoma multiforme cases unmethylated at MGMT promoter express different levels of MGMT protein; (d) anaplastic oligodendroglioma methylated at MGMT promoter demonstrates loss of MGMT protein expression (all $\times 40$ ).

of gene silencing, and partial methylation might account for protein expression in spite of evidence of methylated MGMT promoter.

The MSP protocol itself presented some technical challenges during the initial validation steps. As a result of tissue necrosis, and the infiltrating growth pattern of gliomas, low DNA yields were initially recovered on a number of specimens. MGMT MSP testing needed to be repeated for seven of the specimens, because insufficient DNA was isolated from initial tissue sections. Subsequent sections yielded enough DNA for testing to be completed successfully. MGMT MSP was unsuccessful, even after repeat testing, with three specimens (two GBM specimens and one astrocytoma specimen) due to extensive tissue necrosis. In successfully evaluated tumors, cutting thicker sections and selecting tissue blocks with greatest amount of tumor involvement tended to improve the yield of amplifiable DNA. Loss of DNA during bisulfite treatment was another technical challenge, which prompted us to evaluate several different protocols.
The protocol yielding the best recovery of amplifiable DNA was a commercial DNA Methylation kit (Zymo Research). In order to avoid false negative results, which may be due to several reasons including recovery of partially sectioned cell nuclei, incomplete DNA modification during bisulfite treatment, or loss of DNA during the testing procedure, we selected appropriate quality control measures to assure that all assay steps were well controlled. Incorporating positive methylated DNA and negative unmethylated DNA controls in parallel with patient specimens during the bisulfite reaction and PCR amplification steps gave us assurance that optimal conditions were maintained during all testing steps.

In summary, this simplified protocol allowed us to assess MGMT promoter methylation status of 164 samples out of 167 brain tumor resection specimens submitted for MGMT MSP testing. Since archival tissue proved to be adequate for this testing, the protocol could be easily incorporated into our routine surgical pathology practice. 
1. Hulleman E, Helin K. Molecular mechanisms in gliomagenesis. Adv Cancer Res 2005;94:1-21.

2. Andesson U, Malmer B, Bergenheim AT, et al. Heterogeneity in the expression markers of drug resistance in brain tumors. Clin Neuropathol 2004;23:21-27.

3. Nieder C, Adam M, Grosu AL. Combined modality treatment of glioblastoma multiforme: the role of Temozolomide. Rev Recent Clin Trials 2006;1:43-51.

4. Hegi ME, Diserens A-C, Gorlia T, et al. MGMT gene silencing and benefit from Temozolomide in glioblastomas. N Engl J Med 2005;352:997-1003.

5. Stupp R, Dietrich P-Y, Kraljevic SO. Promising survival for patients with newly diagnosed glioblastoma multiforme treated with concomitant radiation plus Temozolomide followed by adjuvant Temozolomide. J Clin Oncol 2002;20:1375-1382.

6. Friedman HS, Kerby $\mathrm{T}$, Calvert $\mathrm{H}$. Temozolomide and treatment of malignant glioma. Clin Cancer Res 2000;6:2585-2597.

7. Kaina B, Christmann M. DNA repair in resistance to alkylating anticancer drugs. Int J Clin Pharmacol Ther 2002;40:354-367.

8. Gerson SL. MGMT: its role in cancer etiology and cancer therapeutics. Nat Rev Cancer 2004;4:296-307.

9. Esteller $M$, Herman JG. Generating mutations but providing chemosensitivity: the role of $\mathrm{O}^{6}$-methylguanine DNA methyltransferase in human cancer. Oncogene 2004;23:1-8.

10. Jaeckie KA, Eyre HJ, Townsend JJ, et al. Correlation of $\mathrm{O}^{6}$ methylguanine-DNA methyltransferase levels with survival of malignant astrocytoma patients treated with bischloroethylnitrosourea: a Southwest Oncology Group Study. J Clin Oncol 1998;16:3310-3315.

11. Hegi ME, Diserens A-C, Godard S, et al. Clinical trial substantiates the predictive value of 0-6-methylguanine-DNA methyltransferase promoter methylation in glioblastoma patients treated with temozolomide. Clin Cancer Res 2004;10:1871-1874.

12. Esteller M, Garcia-Foncillas J, Andion E, et al. Inactivation of the DNArepair gene $M G M T$ and the clinical response of gliomas to alkylating agents. N Engl J Med 2000;343:1350-1354.

13. Brell $M$, Tortosa $A$, Verger $E$, et al. Prognostic significance of $O^{6}$ methylguanine-DNA methyltransferase determined by promoter hypermethylation and immunohistochemical expression in anaplastic gliomas. Cin Cancer Res 2005;11:5167-5174.

14. Herman JG, Baylin SB. Gene silencing in cancer in association with promoter hypermethylation. N Engl J Med 2003;349:2042-2054.
15. Jones PA, Laird PW. Cancer epigenetics comes of age. Nat Genet 1999;21:163-167.

16. Baylin SB, Herman JG. DNA hypermethylation in tumorigenesis: epigenetics joins genetics. Trends Genet 2000;16:168-174.

17. Costello JE, Fruhwald MC, Smiraglia DJ, et al. Aberrant CpG-island methylation has nonrandom and tumour-type-specific patterns. Nat Genet 2000;24:132-138.

18. Merlo A, Herman JG, Mao L, et al. $5^{\prime}$ CpG island methylation is associated with transcriptional silencing of the tumor suppressor p16/CDKN2/MTS1 in human cancers. Nat Med 1995;1:686-692.

19. Herman JG, Jen J, Merlo A, et al. Hypermethylation-associated inactivation indicates a tumor suppressor role for $\mathrm{p} 15^{\mathrm{INK} 4 \mathrm{~B}}$. Cancer Res 1996;56:722-727.

20. Esteller $M$, Hamilton SR, Burger $P C$, et al. Inactivation of the DNA repair gene 0-6-methylguanine-DNA methyltransferase by promoter hypermethylation is a common event in primary human neoplasia. Cancer Res 1999;59:793-797.

21. Rein $\mathrm{T}$, DePamphilis ML, Zorbas H. Identifying 5-methylcytosine and related modifications in DNA genomes. Nucleic Acids Res 1998:26:2255-2264.

22. Gonzalez-Gomez P, Bello MJ, Arjona D, et al. Promoter hypermethylation of multiple genes in astrocytic gliomas. Int J Oncol 2003;22:601-608.

23. Zochbauer-Muller S, Fong KM, Virmani AK, et al. Aberrant promoter methylation of multiple genes in non-small cell lung cancers. Cancer Res 2001;61:249-255.

24. Tanaka S, Kobayahi I, Utsuki $\mathrm{S}$, et al. $0^{6}$ methylguanine-DNA methyltransferase gene expression in gliomas by means of real-time quantitative RT-PCR and clinical response to nitrosoureas. Int J Cancer 2003;103:67-72.

25. Rolhion $\mathrm{C}$, Penault-Llorca $\mathrm{F}$, Kemeny $\mathrm{JL}$, et al. $\mathrm{O}^{6}$ methylguanine-DNA methyltransferase gene (MGMT) expression in human glioblastomas in relation to patient characteristics and p53 accumulation. Int J Cancer 1999;416:416-420

26. Möllemann $M$, Wolter $M$, Felsberg J, et al. Frequent promoter hypermethylation and low expression of the MGMT gene in oligodendroglial tumors. Int J Cancer 2005;113:379-385.

27. Ogino S, Kawasaki T, Brahmandam M, et al. Precision and performance characteristics of bisulfite conversion and real-time PCR (Mehtyl Light) for quantitative DNA methylation analysis. J Mol Diagnostics 2006;8:209-217. 